\title{
Double-Peakon Solutions of Two Four-Component Camassa-Holm Type Equations
}

\author{
Yuanli Li, Qilao Zha \\ College of Mathematics Science, Inner Mongolia Normal University, Hohhot, China \\ Email: 1416528264@qq.com,zhaqilao@imnu.edu.cn
}

Received 6 May 2016; accepted 12 July 2016; published 15 July 2016

\begin{abstract}
This paper is contributed to study two new integrable four-component systems reduced from a multi-component generation of Camassa-Holm equation. Some double peakon solutions of both systems are described in an explicit formula by the method of variation of constant for ordinary differential equations. These double peakon solutions are established in weak sense. The dynamic behaviors of the obtained double peakon solutions are illustrated by some figures.
\end{abstract}

\section{Keywords}

\section{Peakon Solution, Dynamic Behavior, Four-Component CH Type Equation}

\section{Introduction}

In 1993, Camassa and Holm derived the celebrated Camassa-Holm (CH) equation [1], which is formally integrable, since it admits Lax pair formalism [1], bi-Hamiltonian structure [2] as well as infinitely many conservation laws [2]. One of the remarkable properties of the equation is that it possesses peakon wave solutions. Subsequently, a large amount of literature was devoted to find new integrable models with peakon solutions, such as Degasperis-Procesi (DP) equation, the Fokas-Olver-Rosenau-Qiao (FORQ) equation, the Novikov's cubic nonlinear equation and some other completely integrable peakon systems. It is a natural idea to continue studying multi-component generalizations of peakon equations. One of the most popular two-component integrable systems, which admits Lax Pair and infinitely conservation laws, has multi-peakon solitons [3]. A three-component model with peakon solutions has been studied by Geng and Xue [4].

Very recently, in Ref. [5], another multi-component system of Camassa-Holm equation, which admited Lax pair and infinitely many conservation laws, denoted by $\mathrm{CH}(\mathrm{N}, \mathrm{H})$ with $2 \mathrm{~N}$ components and an arbitrary smooth function $H$ of $u_{j}, v_{j}(1 \leq j \leq N)$ and their derivatives, was derived and studied

$$
m_{j, t}=\left(m_{j} H\right)_{x}+m_{j} H+\frac{1}{(N+1)^{2}} \sum_{i=1}^{N}\left[m_{i}\left(u_{j}-u_{j, x}\right)\left(v_{i}+v_{i, x}\right)+m_{j}\left(u_{i}-u_{i, x}\right)\left(v_{i}+v_{i, x}\right)\right]
$$




$$
\begin{gathered}
n_{j, t}=\left(n_{j} H\right)_{x}-n_{j} H-\frac{1}{(N+1)^{2}} \sum_{i=1}^{N}\left[n_{i}\left(u_{i}-u_{i, x}\right)\left(v_{j}+v_{j, x}\right)+n_{j}\left(u_{i}-u_{i, x}\right)\left(v_{i}+v_{i, x}\right)\right], \\
m_{j}=u_{j}-u_{j, x x}, n_{j}=v_{j}-v_{j, x x}, 1 \leq j \leq N .
\end{gathered}
$$

Particularly, in the case of $H=0, N=2$, Equation (1) becomes the following system

$$
\begin{aligned}
& m_{1 t}=\frac{1}{9}\left\{m_{1}\left[2\left(u_{1}-u_{1, x}\right)\left(v_{1}+v_{1, x}\right)+\left(u_{2}-u_{2, x}\right)\left(v_{2}+v_{2, x}\right)\right]+m_{2}\left(u_{1}-u_{1, x}\right)\left(v_{2}+v_{2, x}\right)\right\}, \\
& m_{2 t}=\frac{1}{9}\left\{m_{1}\left(u_{2}-u_{2, x}\right)\left(v_{1}+v_{1, x}\right)+m_{2}\left[\left(u_{1}-u_{1, x}\right)\left(v_{1}+v_{1, x}\right)+2\left(u_{2}-u_{2, x}\right)\left(v_{2}+v_{2, x}\right)\right]\right\}, \\
& n_{1 t}=-\frac{1}{9}\left\{n_{1}\left[2\left(u_{1}-u_{1, x}\right)\left(v_{1}+v_{1, x}\right)+\left(u_{2}-u_{2, x}\right)\left(v_{2}+v_{2, x}\right)\right]+n_{2}\left(u_{2}-u_{2, x}\right)\left(v_{1}+v_{1, x}\right)\right\}, \\
& n_{2 t}=-\frac{1}{9}\left\{n_{1}\left(u_{1}-u_{1, x}\right)\left(v_{2}+v_{2, x}\right)+n_{2}\left[\left(u_{1}-u_{1, x}\right)\left(v_{1}+v_{1, x}\right)+2\left(u_{2}-u_{2, x}\right)\left(v_{2}+v_{2, x}\right)\right]\right\}, \\
& m_{1}=u_{1}-u_{1, x x}, m_{2}=u_{2}-u_{2, x x}, n_{1}=v_{1}-v_{1, x x}, n_{2}=v_{2}-v_{2, x x},
\end{aligned}
$$

and in the case of $H=-\frac{1}{9}\left[\left(u_{1}-u_{1, x}\right)\left(v_{1}+v_{1, x}\right)+\left(u_{2}-u_{2, x}\right)\left(v_{2}+v_{2, x}\right)\right], N=2$, it is reduced into

$$
\begin{aligned}
& m_{1 t}=\left(m_{1} H\right)_{x}+\frac{1}{9} m_{1}\left(u_{1}-u_{1, x}\right)\left(v_{1}+v_{1, x}\right)+\frac{1}{9} m_{2}\left(u_{1}-u_{1, x}\right)\left(v_{2}+v_{2, x}\right), \\
& m_{2 t}=\left(m_{2} H\right)_{x}+\frac{1}{9} m_{1}\left(u_{2}-u_{2, x}\right)\left(v_{1}+v_{1, x}\right)+\frac{1}{9} m_{2}\left(u_{2}-u_{2, x}\right)\left(v_{2}+v_{2, x}\right), \\
& n_{1 t}=\left(n_{1} H\right)_{x}-\frac{1}{9} n_{1}\left(u_{1}-u_{1, x}\right)\left(v_{1}+v_{1, x}\right)-\frac{1}{9} n_{2}\left(u_{2}-u_{2, x}\right)\left(v_{1}+v_{1, x}\right), \\
& n_{2 t}=\left(n_{2} H\right)_{x}-\frac{1}{9} n_{1}\left(u_{1}-u_{1, x}\right)\left(v_{2}+v_{2, x}\right)-\frac{1}{9} n_{2}\left(u_{2}-u_{2, x}\right)\left(v_{2}+v_{2, x}\right), \\
& m_{1}=u_{1}-u_{1, x x}, m_{2}=u_{2}-u_{2, x x}, n_{1}=v_{1}-v_{1, x x}, n_{2}=v_{2}-v_{2, x x} .
\end{aligned}
$$

Xia and Qiao have presented bi-Hamiltonian structures and single peakon solutions [5] of Equations (2) and (3). According to the work in [5], we will investigate the double peakon solutions of Equations (2) and (3) in this paper. Section 2 is devoted to look for double peakon solutions of Equations (2) and (3). Further, we discuss the dynamic behaviors of the obtained peakon solutions by some figures. Some conclusions and open problems are addressed in Section 3.

\section{Two-Peakon Solutions}

\subsection{Two-Peakon Solutions to Equation (2)}

We assume that the system Equation (2) admits two peakon solitons of the form

$$
\begin{aligned}
& u_{1}=p_{1} \mathrm{e}^{-\left|x-q_{1}\right|}+p_{2} \mathrm{e}^{-\left|x-q_{2}\right|}, u_{2}=r_{1} \mathrm{e}^{-\left|x-q_{1}\right|}+r_{2} \mathrm{e}^{-\left|x-q_{2}\right|}, \\
& v_{1}=s_{1} \mathrm{e}^{-\left|x-q_{1}\right|}+s_{2} \mathrm{e}^{-\left|x-q_{2}\right|}, v_{2}=w_{1} \mathrm{e}^{-\left|x-q_{1}\right|}+w_{2} \mathrm{e}^{-\left|x-q_{2}\right|},
\end{aligned}
$$

where $p_{i}(t), r_{i}(t), s_{i}(t), w_{i}(t)$ and $q_{i}(t) \quad(i=1,2)$, are functions of $t$ to be determined. Moreover, we can obtain their derivatives in the weak sense as follows

$$
\begin{aligned}
& u_{1, x}=-p_{1} \operatorname{sgn}\left(x-q_{1}\right) \mathrm{e}^{-\left|x-q_{1}\right|}-p_{2} \operatorname{sgn}\left(x-q_{2}\right) \mathrm{e}^{-\left|x-q_{2}\right|}, \\
& u_{2, x}=-r_{1} \operatorname{sgn}\left(x-q_{1}\right) \mathrm{e}^{-\left|x-q_{1}\right|}-r_{2} \operatorname{sgn}\left(x-q_{2}\right) \mathrm{e}^{-\left|x-q_{2}\right|}, \\
& v_{1, x}=-s_{1} \operatorname{sgn}\left(x-q_{1}\right) \mathrm{e}^{-\left|x-q_{1}\right|}-s_{2} \operatorname{sgn}\left(x-q_{2}\right) \mathrm{e}^{-\left|x-q_{2}\right|}, \\
& v_{2, x}=-w_{1} \operatorname{sgn}\left(x-q_{1}\right) \mathrm{e}^{-\left|x-q_{1}\right|}-w_{2} \operatorname{sgn}\left(x-q_{2}\right) \mathrm{e}^{-\left|x-q_{2}\right|}, \\
& m_{1}=2 p_{1} \delta\left(x-q_{1}\right)+2 p_{2} \delta\left(x-q_{2}\right), m_{2}=2 r_{1} \delta\left(x-q_{1}\right)+2 r_{2} \delta\left(x-q_{2}\right),
\end{aligned}
$$




$$
n_{1}=2 s_{1} \delta\left(x-q_{1}\right)+2 s_{2} \delta\left(x-q_{2}\right), n_{2}=2 w_{1} \delta\left(x-q_{1}\right)+2 w_{2} \delta\left(x-q_{2}\right) .
$$

Without loss of generality, we assume that $q_{1}>q_{2}$. Substituting Equations (4) and (5) into Equation (2) and in the distribution sense, we can obtain the following double peakon differential equations

$$
\begin{aligned}
& q_{1, t}=q_{2, t}=0, \\
& p_{1, t}=\frac{4}{27} p_{1} \Delta_{11}+\frac{2}{9} p_{1} \Delta_{21} e^{q_{2}-q_{1}}+\frac{2}{9} w_{1} e^{q_{2}-q_{1}} \Delta, \quad p_{2, t}=\frac{4}{27} p_{2} \Delta_{22}+\frac{2}{9} p_{2} \Delta_{21} e^{q_{2}-q_{1}}, \\
& r_{1, t}=\frac{4}{27} r_{1} \Delta_{11}+\frac{2}{9} r_{1} \Delta_{21} e^{q_{2}-q_{1}}-\frac{2}{9} s_{1} e^{q_{2}-q_{1}} \Delta, \quad r_{2, t}=\frac{4}{27} r_{2} \Delta_{22}+\frac{2}{9} r_{2} \Delta_{21} e^{q_{2}-q_{1}}, \\
& s_{1, t}=-\frac{4}{27} s_{1} \Delta_{11}-\frac{2}{9} s_{1} \Delta_{21} e^{q_{2}-q_{1}}, \quad s_{2, t}=-\frac{4}{27} s_{2} \Delta_{22}-\frac{2}{9} s_{2} \Delta_{21} e^{q_{2}-q_{1}}-\frac{2}{9} r_{2} e^{q_{2}-q_{1}} \Lambda, \\
& w_{1, t}=-\frac{4}{27} w_{1} \Delta_{11}-\frac{2}{9} w_{1} \Delta_{21} e^{q_{2}-q_{1}}, \quad w_{2, t}=-\frac{4}{27} w_{2} \Delta_{22}-\frac{2}{9} w_{2} \Delta_{21} e^{q_{2}-q_{1}}+\frac{2}{9} p_{2} e^{q_{2}-q_{1}} \Lambda,
\end{aligned}
$$

where $\Delta_{11}=p_{1} s_{1}+r_{1} w_{1}, \Delta_{22}=p_{2} s_{2}+r_{2} w_{2}, \quad \Delta_{21}=2\left(p_{2} s_{1}+r_{2} w_{1}\right), \Delta=r_{1} p_{2}-p_{1} r_{2}$ and $\Lambda=s_{1} w_{2}-s_{2} w_{1} . \Delta_{11}$ and $\Delta_{22}$ taking derivative with respect to $t$, we can obtain

$$
\Delta_{11, t}=0, \quad \Delta_{22, t}=0
$$

Thus we have

$$
\Delta_{11}=A_{1}, \quad \Delta_{22}=B_{1},
$$

where $A_{1}$ and $B_{1}$ are arbitrary integration constants. In the following, we assume that $A_{1}=B_{1}$.

$\Delta_{21}$ taking derivative with respect to $t$ and combining with Equation (6), we see

$$
\Delta_{21, t}=0 \text {. }
$$

Thus, we can get

$$
\Delta_{21}=C_{1}
$$

where $C_{1}$ is arbitrary constant.

Combining the Equation (6) with Equations (8) and (10), Equation (6) is reduced to

$$
\begin{aligned}
& q_{1}=m \quad q_{2}=n, \\
& p_{1, t}=\frac{4}{27} p_{1} A_{1}+\frac{2}{9} p_{1} C_{1} e^{n-m}+\frac{2}{9} w_{1} e^{n-m} \Delta, \quad p_{2, t}=\frac{4}{27} p_{2} A_{1}+\frac{2}{9} p_{2} C_{1} e^{n-m}, \\
& r_{1, t}=\frac{4}{27} r_{1} A_{1}+\frac{2}{9} r_{1} C_{1} e^{n-m}-\frac{2}{9} s_{1} e^{n-m} \Delta, \quad r_{2, t}=\frac{4}{27} r_{2} A_{1}+\frac{2}{9} r_{2} C_{1} e^{n-m}, \\
& s_{1, t}=-\frac{4}{27} s_{1} A_{1}-\frac{2}{9} s_{1} C_{1} e^{n-m}, \quad s_{2, t}=-\frac{4}{27} s_{2} A_{1}-\frac{2}{9} s_{2} C_{1} e^{n-m}-\frac{2}{9} r_{2} e^{n-m} \Lambda, \\
& w_{1, t}=-\frac{4}{27} w_{1} A_{1}-\frac{2}{9} w_{1} C_{1} e^{n-m}, \quad w_{2, t}=-\frac{4}{27} w_{2} A_{1}-\frac{2}{9} w_{2} C_{1} e^{n-m}+\frac{2}{9} p_{2} e^{n-m} \Lambda,
\end{aligned}
$$

where $m, n$ are integration constants. According to Equation (11), we can arrive at

$$
\begin{aligned}
& p_{2}=C_{2} e^{\left(\frac{4}{27} A_{1}+\frac{2}{9} C_{1} e^{n-m}\right) t}, r_{2}=C_{3} e^{\left(\frac{4}{27} A_{1}+\frac{2}{9} C_{1} e^{n-m}\right) t}, \\
& S_{1}=C_{4} e^{-\left(\frac{4}{27} A_{1}+\frac{2}{9} C_{1} e^{n-m}\right) t}, \quad w_{1}=C_{5} e^{-\left(\frac{4}{27} A_{1}+\frac{2}{9} C_{1} e^{n-m}\right) t},
\end{aligned}
$$

where $C_{2}, C_{3}, C_{4}$ and $C_{5}$ denote integration constants. Moreover, with the help of Equation (11), we easily obtain that

$$
\begin{aligned}
& \Delta_{t}=\left(\frac{8}{27} A_{1}+\frac{4}{9} C_{1} e^{n-m}\right) \Delta-\frac{2}{9}\left(C_{3} C_{5}+C_{2} C_{4}\right) e^{n-m} \Delta, \\
& \Lambda_{t}=-\left(\frac{8}{27} A_{1}+\frac{4}{9} C_{1} e^{n-m}\right) \Lambda+\frac{2}{9}\left(C_{3} C_{5}+C_{2} C_{4}\right) e^{n-m} \Lambda .
\end{aligned}
$$


Solving the differential equations of Equation (13), we get

$$
\begin{aligned}
& \Delta=C_{6} e^{\left(\frac{8}{27} A_{1}+\frac{4}{9} C_{1} e^{n-m}-\frac{2}{9} C_{2} C_{4} e^{n-m}-\frac{2}{9} C_{3} C_{5} e^{n-m}\right) t}, \\
& \Lambda=C_{7} e^{-\left(\frac{8}{27} A_{1}+\frac{4}{9} C_{1} e^{n-m}-\frac{2}{9} C_{2} C_{4} e^{n-m}-\frac{2}{9} C_{3} C_{5} e^{n-m}\right) t} .
\end{aligned}
$$

Using the method of variation of constant, we can have

$$
\begin{aligned}
& p_{1}=\left[-\frac{C_{5} C_{6}}{C_{2} C_{4}+C_{3} C_{5}} e^{-\frac{2}{9}\left(C_{2} C_{4}+C_{3} C_{5}\right) e^{n-m} t}+C_{8}\right] e^{\left(\frac{4}{27} A_{1}+\frac{2}{9} C_{1} e^{n-m}\right) t}, \\
& r_{1}=\left[\frac{C_{4} C_{6}}{C_{2} C_{4}+C_{3} C_{5}} e^{-\frac{2}{9}\left(C_{2} C_{4}+C_{3} C_{5}\right) e^{n-m} t}+C_{9}\right] e^{\left(\frac{4}{27} A_{1}+\frac{2}{9} C_{1} e^{n-m}\right) t}, \\
& s_{2}=\left[-\frac{C_{3} C_{7}}{C_{2} C_{4}+C_{3} C_{5}} e^{\frac{2}{9}\left(C_{2} C_{4}+C_{3} C_{5}\right) e^{n-m_{t}}}+C_{10}\right] e^{-\left(\frac{4}{27} A_{1}+\frac{2}{9} C_{1} e^{n-m}\right) t}, \\
& w_{2}=\left[\frac{C_{2} C_{7}}{C_{2} C_{4}+C_{3} C_{5}} e^{\frac{2}{9}\left(C_{2} C_{4}+C_{3} C_{5}\right) e^{n-m} t}+C_{11}\right] e^{-\left(\frac{4}{27} A_{1}+\frac{2}{9} C_{1} e^{n-m}\right) t} .
\end{aligned}
$$

Based on Equations (8) and (10), those constants have relations as

$$
C_{2} C_{4}+C_{3} C_{5}=\frac{C_{1}}{2}, C_{2} C_{10}+C_{3} C_{11}=C_{8} C_{4}+C_{9} C_{5}=A_{1} .
$$

Thus, we establish the double peakon solutions of the Equation (2)

$$
\begin{aligned}
u_{1}= & {\left[-\frac{C_{5} C_{6}}{C_{2} C_{4}+C_{3} C_{5}} e^{-\frac{2}{9}\left(C_{2} C_{4}+C_{3} C_{5}\right) e^{n-m} t}+C_{8}\right] e^{\left(\frac{4}{27} A_{1}+\frac{2}{9} C_{1} e^{n-m}\right) t} e^{-|x-m|} } \\
& +C_{2} e^{\left(\frac{4}{27} A_{1}+\frac{2}{9} C_{1} e^{n-m}\right) t} e^{-|x-n|}, \\
u_{2}= & {\left[\frac{C_{4} C_{6}}{C_{2} C_{4}+C_{3} C_{5}} e^{-\frac{2}{9}\left(C_{2} C_{4}+C_{3} C_{5}\right) e^{n-m} t}+C_{9}\right] e^{\left(\frac{4}{27} A_{1}+\frac{2}{9} C_{1} e^{n-m}\right) t} e^{-|x-m|} } \\
& +C_{3} e^{\left(\frac{4}{27} A_{1}+\frac{2}{9} C_{1} e^{n-m}\right) t} e^{-|x-n|}, \\
v_{1}= & C_{4} e^{-\left(\frac{4}{27} A_{1}+\frac{2}{9} C_{1} e^{n-m}\right) t} e^{-|x-m|} \\
& +\left[-\frac{C_{3} C_{7}}{C_{2} C_{4}+C_{3} C_{5}} e^{\frac{2}{9}\left(C_{2} C_{4}+C_{3} C_{5}\right) e^{n-m} t}+C_{10}\right] e^{-\left(\frac{4}{27} A_{1}+\frac{2}{9} C_{1} e^{n-m}\right) t} e^{-|x-n|}, \\
v_{2}= & C_{5} e^{-\left(\frac{4}{27} A_{1}+\frac{2}{9} C_{1} e^{n-m}\right) t} e^{-|x-m|} \\
& +\left[\frac{C_{2} C_{7}}{C_{2} C_{4}+C_{3} C_{5}} e^{\frac{2}{9}\left(C_{2} C_{4}+C_{3} C_{5}\right) e^{n-m} t}+C_{11}\right] e^{-\left(\frac{4}{27} A_{1}+\frac{2}{9} C_{1} e^{n-m}\right) t} e^{-|x-n|},
\end{aligned}
$$

where $C_{i}$ and $A_{1}(i=1 \cdots 11)$ satisfy Equation (16).

Figure 1 show the profiles of the double peakon solutions Equation (17). The amplitudes of the peakons grow or decay exponentially with time $t$. All peak positions don't change along with the time $t$ and the collision between the two-peakon waves will never happen.

\subsection{Double Peakon Solutions to Equation (3)}

By means of the similar calculation as those in the Section 2.1, taking $q_{1}>q_{2}$, we arrive at the differential equations as follows 

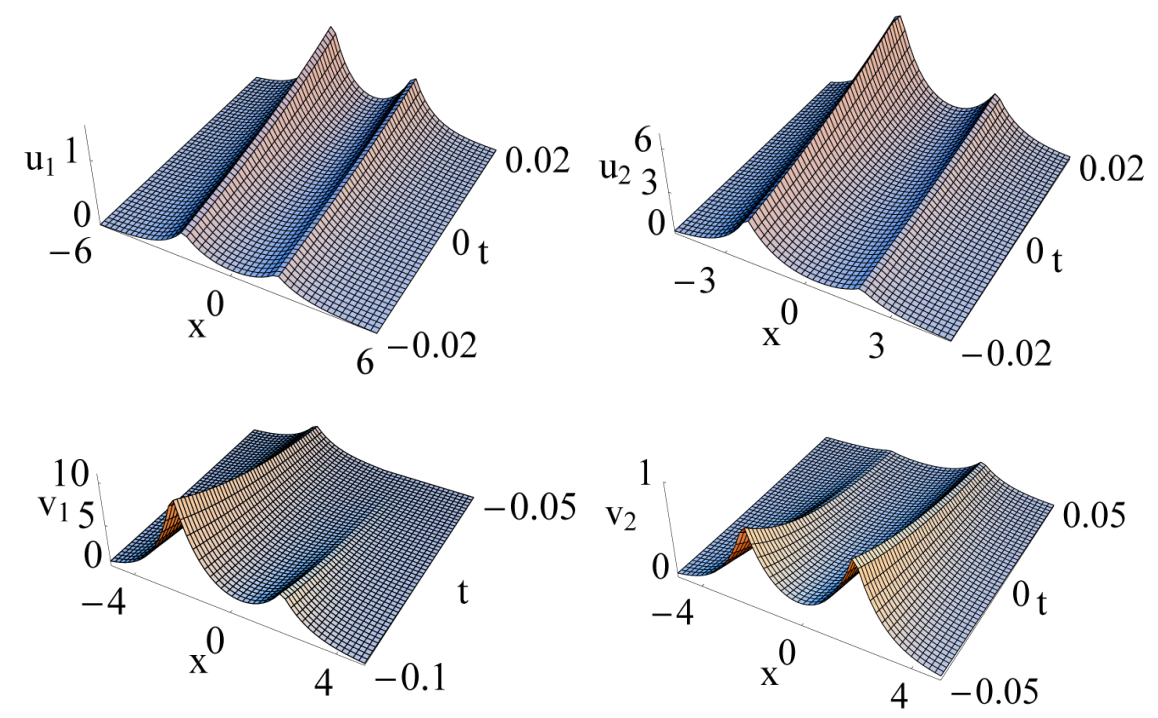

Figure 1. 3D graphs of the double peakon solutions for $u_{i}, v_{i}, i=1,2$, defined by Equation (17) with $C_{1}=2, C_{2}=1 / 2, C_{3}=2, C_{4}=1, C_{5}=1 / 4, C_{6}=1, C_{7}=1, C_{8}=1, C_{9}=4, C_{10}=1, C_{11}=0, A_{1}=2, m=-2, n=2$.

$$
\begin{aligned}
& q_{1, t}=\frac{2}{27} a_{1}+\frac{2}{9}\left(p_{2} s_{1}+r_{2} w_{1}\right) e^{-\left|q_{1}-q_{2}\right|}, \quad q_{2, t}=\frac{2}{27} b_{1}+\frac{2}{9}\left(p_{2} s_{1}+r_{2} w_{1}\right) e^{-\left|q_{1}-q_{2}\right|} \\
& p_{1, t}=\frac{2}{27} p_{1} a_{1}+\frac{2}{9} p_{2} a_{1} e^{-\left|q_{1}-q_{2}\right|}, \quad p_{2, t}=\frac{2}{27} p_{2} b_{1}+\frac{2}{9} p_{2}\left(p_{2} s_{1}+r_{2} w_{1}\right) e^{-\left|q_{1}-q_{2}\right|} \\
& r_{1, t}=\frac{2}{27} r_{1} a_{1}+\frac{2}{9} r_{2} a_{1} e^{-\left|q_{1}-q_{2}\right|}, \quad r_{2, t}=\frac{2}{27} r_{2} b_{1}+\frac{2}{9} r_{2}\left(p_{2} s_{1}+r_{2} w_{1}\right) e^{-\left|q_{1}-q_{2}\right|} \\
& s_{1, t}=-\frac{2}{27} s_{1} a_{1}-\frac{2}{9} s_{1}\left(p_{2} s_{1}+r_{2} w_{1}\right) e^{-\left|q_{1}-q_{2}\right|}, \quad s_{2, t}=-\frac{2}{27} s_{2} b_{1}-\frac{2}{9} s_{1} b_{1} e^{-\left|q_{1}-q_{2}\right|} \\
& w_{1, t}=-\frac{2}{27} w_{1} a_{1}-\frac{2}{9} w_{1}\left(p_{2} s_{1}+r_{2} w_{1}\right) e^{-\left|q_{1}-q_{2}\right|}, \quad w_{2, t}=-\frac{2}{27} w_{2} b_{1}-\frac{2}{9} w_{1} b_{1} e^{-\left|q_{1}-q_{2}\right|}
\end{aligned}
$$

where $a_{1}$ and $b_{1}$ are constants. From which, it is easy to see that we may have the following relations

$$
\begin{aligned}
& \frac{w_{1}}{s_{1}}=C_{1}, \quad \frac{p_{2}}{r_{2}}=C_{2}, \quad \frac{p_{2, t}}{p_{2}}=q_{2, t}, \\
& \frac{s_{1, t}}{s_{1}}=-q_{1, t}, \quad q_{1}-q_{2}=\frac{2}{27}\left(a_{1}-b_{1}\right) t+C_{4} .
\end{aligned}
$$

Supposing that $a_{1}=b_{1}$ and letting $\Omega_{21}=p_{2} s_{1}+r_{2} w_{1}$, we can readily see $\Omega_{21, t}=0, \Omega_{21}=C_{3}$. Thus, the $t$-dependent functions satisfy the following ordinary differential equations

$$
\begin{aligned}
q_{1, t} & =\frac{2}{27} a_{1}+\frac{2}{9} C_{3} e^{-C_{4}}, & q_{2, t} & =\frac{2}{27} a_{1}+\frac{2}{9} C_{3} e^{-C_{4}}, \\
p_{1, t} & =\frac{2}{27} p_{1} a_{1}+\frac{2}{9} p_{2} a_{1} e^{-C_{4}}, & p_{2, t} & =\frac{2}{27} p_{2} a_{1}+\frac{2}{9} C_{3} p_{2} e^{-C_{4}}, \\
r_{1, t} & =\frac{2}{27} r_{1} a_{1}+\frac{2}{9} r_{2} a_{1} e^{-C_{4}}, & r_{2, t} & =\frac{2}{27} r_{2} a_{1}+\frac{2}{9} r_{2} C_{3} e^{-C_{4}}, \\
s_{1, t} & =-\frac{2}{27} s_{1} a_{1}-\frac{2}{9} s_{1} C_{3} e^{-C_{4}}, & s_{2, t} & =-\frac{2}{27} s_{2} a_{1}-\frac{2}{9} s_{1} a_{1} e^{-C_{4}}, \\
w_{1, t} & =-\frac{2}{27} w_{1} a_{1}-\frac{2}{9} w_{1} C_{3} e^{-C_{4}}, & w_{2, t} & =-\frac{2}{27} w_{2} a_{1}-\frac{2}{9} w_{1} a_{1} e^{-C_{4}} .
\end{aligned}
$$


Thus, we obtain the double peakon solutions of Equation (3)

$$
\begin{aligned}
& u_{1}=\left[\frac{C_{6} a_{1}}{C_{3}} e^{\frac{2}{9} C_{3^{2}} e^{-C_{4} t}}+C_{8}\right] e^{\frac{2}{27} a_{1} t} e^{-\mid x-\frac{2}{27} a_{1}-\frac{2}{9} C_{3} e^{-C_{4} t-C_{5} \mid}} \\
& +C_{6} e^{\left(\frac{2}{27} a_{1}+\frac{2}{9} C_{3} e^{-C_{4}}\right) t} e^{-\mid x-\frac{2}{27} a_{1}-\frac{2}{9} C_{3} e^{-C_{4} t-C_{5}+C_{4} \mid}}, \\
& u_{2}=\left[\frac{C_{6} a_{1}}{C_{2} C_{3}} e^{\frac{2}{9} C_{3} e^{-C_{4} t}}+C_{9}\right] e^{\frac{2}{27} a_{1} t} e^{-\mid x-\frac{2}{27} a_{1}-\frac{2}{9} C_{3} e^{-C_{4 t}-C_{5} \mid}} \\
& +\frac{C_{6}}{C_{2}} e^{\left(\frac{2}{27} a_{1}+\frac{2}{9} C_{3} e^{-C_{4}}\right) t} e^{-\mid x-\frac{2}{27} a_{1}-\frac{2}{9} C_{3} e^{-C_{4} t-C_{5}+C_{4} \mid}}, \\
& v_{1}=C_{7} e^{-\left(\frac{2}{27} a_{1}+\frac{2}{9} C_{3} e^{-C_{4}}\right) t} e^{-\mid x-\frac{2}{27} a_{1} t-\frac{2}{9} C_{3} e^{-C_{4} t-C_{5} \mid}} \\
& +\left[\frac{C_{7} a_{1}}{C_{3}} e^{-\frac{2}{9} C_{3} e^{-C_{4} t}}+C_{10}\right] e^{-\frac{2}{27} a_{1 t}} e^{-\mid x-\frac{2}{27} a_{1}-\frac{2}{9} C_{3} e^{-C_{4}} C_{t-C_{5}+C_{4} \mid}}, \\
& V_{2}=C_{7} C_{1} e^{-\left(\frac{2}{27} a_{1}+\frac{2}{9} C_{3} e^{-C_{4}}\right) t} e^{-\mid x-\frac{2}{27} a_{1}-\frac{2}{9} C_{3} e^{-C_{4 t}-C_{5} \mid}} \\
& +\left[\frac{C_{7} C_{1} a_{1}}{C_{3}} e^{-\frac{2}{9} C_{3} e^{-C_{4} t}}+C_{11}\right] e^{-\frac{2}{27} a_{1} t} e^{-\mid x-\frac{2}{27} a_{1} t-\frac{2}{9} C_{3} e^{-C_{4} t-C_{5}+C_{4} \mid},}
\end{aligned}
$$

where $C_{2}=C_{6}, C_{6} C_{7}+C_{7} C_{1}=C_{3}, C_{8}+C_{1} C_{9}=0$ and $C_{11}+C_{2} C_{10}=0$.

\section{Conclusion}

We provide an approach to obtain the double peakon solutions for the four-component $\mathrm{CH}$ type Equations (2) and (3) in the case of $\Delta_{11}=\Delta_{22}$ and $a_{1}=b_{1}$ respectively. However, its exact double peakon solutions without $\Delta_{11}=\Delta_{22}$ or $a_{1}=b_{1}$ are expected to attract more endeavor to study.

\section{Acknowledgements}

This work was supported by the National Natural Science Foundation of China under Grant No. 11261037, the Natural Science Foundation of Inner Mongolia Autonomous Region under Grant No. 2014MS0111, the Caoyuan Yingcai Program of Inner Mongolia Autonomous Region under Grant No. CYYC2011050, the Program for Young Talents of Science and Technology in Universities of Inner Mongolia Autonomous Region under Grant No. NJYT14A04.

\section{References}

[1] Camassa, R. and Holm, D.D. (1993) An Integrable Shallow Water Equation with Peaked Solitons. Physical Review Letters, 71, 1661-1664. http://dx.doi.org/10.1103/PhysRevLett.71.1661

[2] Fuchssteiner, B. and Fokas, A.S. (1981) Symplectic Structures, Their Backlund Transformations and Hereditary Symmetries. Physcia D, 4, 47-66. http://dx.doi.org/10.1016/0167-2789(81)90004-X

[3] Xia, B.Q. and Qiao, Z.J. (2015) A Synthetical Two-Component Model with Peakon Solutions. Studies in Applied Mathematics, 135, 248-276. http://dx.doi.org/10.1111/sapm.12085

[4] Geng, X.G. and Xue, B. (2011) A three-Component Generation of Camassa-Holm Equation with N-Peakon Solutions. Advances in Mathematics, 226, 827-839. http://dx.doi.org/10.1016/j.aim.2010.07.009

[5] Xia, B.Q. and Qiao, Z.J. (2015) Multi-Component Generalization of Camassa-Holm Equation. arXiv:1310.0268v2 